\title{
Microstructure of a Paint Primer - a Data-Constrained Modeling Analysis
}

\author{
Sam Yang ${ }^{1, a}$, Dachao Gao ${ }^{1, b}$, Tim Muster ${ }^{1, c}$, Andrew Tulloh ${ }^{1, d}$, \\ Scott Furman ${ }^{1, \mathrm{e}}$, Sherry Mayo ${ }^{1, \mathrm{f}}$, Adrian Trinchi ${ }^{1, g}$ \\ ${ }^{1}$ CSIRO Materials Science \& Engineering, Locked Bag 33, Clayton 3169, Australia \\ aSam.Yang@csiro.au, gao501030@gmail.com, Tim.Muster@csiro.au, 'Andrew.Tulloh@csiro.au \\ 'Scott.Furman@csiro.au, Sherry.Mayo@csiro.au, ${ }^{9}$ Adrian.Trinchi@csiro.au
}

Keywords: Microstructure, corrosion inhibitor, data-constrained modeling

\begin{abstract}
Metallic aerospace components are commonly painted with a primer to improve their corrosion resistance. The primer contains a polymer matrix with embedded corrosion inhibitor and filler particles. Its performance is determined by the microscopic distributions of the particles. Various techniques have been used to quantify such distributions, including X-ray micro-computed tomography (CT). However, its success is sometimes limited by factors such as different particles having similar X-ray $\mathrm{CT}$ absorption properties and their size being smaller than the resolution of micro-CT. In this paper, we have performed two X-ray CT measurements on a paint primer sample consisting of $\mathrm{SrCrO}_{4}$ corrosion inhibitor particles and $\mathrm{UV}$-absorbing $\mathrm{TiO}_{2}$ filler particles. $\mathrm{Fe}$ and $\mathrm{Ti}$ targets were used as X-ray sources with different spectral distributions. The measured CT data sets were used as constraints for a data-constrained microstructure modeling (DCM) prediction of the sample's microscopic structures. DCM model predictions were compared with experimental elemental surface maps and showed reasonable degree of agreement, suggesting X-ray micro-CT combined with DCM modeling would be a powerful technique for detailing the dynamics of chromate-inhibited primers and other multiphase systems where the components are sensitive to incident X-ray energy.
\end{abstract}

\section{Introduction}

One of the most effective means of corrosion protection for metal aerospace components is the use of primers containing hexavalent chromium pigments, such as strontium, calcium and barium chromates [1]. Understanding how hexavalent chromium pigments inhibit corrosion with such high and consistent efficiency is of key importance in corrosion science. Several works have studied these corrosion protection mechanisms, identifying that chromate pigments are slightly soluble in aqueous solution and are transported throughout the organic primer, providing widespread protection for the underlying metal. Less well understood are the dynamic processes controlling inhibitor movement throughout the primer matrix. It is envisaged that by studying the microstructure of such primer coatings, a greater understanding of the corrosion inhibition processes of chromates and other inhibiter molecules may be obtained. In this study, we explore the application of micro-CT to characterize the three-dimensional structure of chromate-inhibited primers. Two X-ray CT measurements, each with a unique beam energy spectrum, were performed on a paint primer sample consisting of strontium chromate $\left(\mathrm{SrCrO}_{4}\right)$ and titania $\left(\mathrm{TiO}_{2}\right)$ particles. The two CT data sets were used as inputs for a data-constrained microstructure modeling (DCM) prediction of the sample microstructure -. Energy Dispersive X-ray Spectroscopy (EDX) was used to characterize the surface of the sample in order to evaluate the ability of the model to predict the 3-dimensional chemical structure of the $\mathrm{SrCrO}_{4}$ and $\mathrm{TiO}_{2}$ throughput the sample.

\section{Sample preparations and X-ray CT}

To make the primer coating, $0.11 \mathrm{~g}$ of strontium chromate (ANAC) and $0.52 \mathrm{~g}$ of titania (Sigma Aldrich) were combined with an epoxy resin. After further processing, small drops of the primer were 
placed onto a Teflon block and dried for one week, forming primer beads that were dissected and mounted onto a thin brass wire holder, suitable for X-ray tomography. The dry primer contained 12.9 $\mathrm{wt} \% \mathrm{SrCrO}_{4}$ and $6.2 \mathrm{wt} \% \mathrm{TiO}_{2}$. Finally, a gold coating was sputtered onto the sample.

$\mathrm{X}$-ray phase-contrast micro-CT was carried out using an X-ray ultra-Microscope (XuM), which adds X-ray phase-contrast micro-CT functionality to an SEM[5]. The electron beam of the SEM is focused onto a target, generating X-rays that pass through the sample to form an image on the X-ray detector. By selecting different metal targets it is possible to modify the dominant wavelength of the $\mathrm{X}$-ray emission. Ti and $\mathrm{Fe}$ were used, the first of which has a characteristic $\mathrm{x}$-ray emission at $4.5 \mathrm{keV}$ and the latter at $6.4 \mathrm{keV}$. The electron beam had an accelerating voltage of $15 \mathrm{kV}$. Furthermore, collecting multiple micro-CT datasets at different wavelengths allows additional information to be obtained, enabling one to distinguish different materials in the sample. For the dataset with the Fe target 720 views were acquired with a 0.5 degree angular step between each view. With the Ti target 360 views with a 0.5 degree step were acquired (due to a data collection problem, however the 180 degree of data is still sufficient for a full 3D reconstruction). The datasets suffered from artifacts arising from misalignment of individual views within each dataset and of one dataset with respect to the other. These were corrected with in-house alignment programs [6]. The views were processed by a phase-retrieval algorithm, converting phase-contrast images into a form suitable for tomographic reconstruction with a conventional CT algorithm [7]. A 3D reconstruction was then generated from each of the datasets, which were later combined for the DCM analysis.

\section{Data-constrained analysis}

The numerical model is implemented on a regular 3D grid of cubic voxels. Information stored at each voxel includes the volume fractions of each material composition and X-ray attenuations for each X-ray beam-energy. The model [4] assumes that the total volume of a voxel is the sum of volumes of individual compositional materials (including void) in the voxel. It also assumes the total attenuation of an X-ray beam by a voxel is the sum over the attenuations by the individual compositional materials in the voxel. The assumptions can be expressed mathematically and have been described in detail elsewhere [4]. Values for the measured total linear absorption coefficients are obtained from the CT data sets [8], and the values for the linear absorption coefficient for each material are available from handbooks of material properties [9]. It is assumed that the beam wavelength takes discrete values $\lambda=\lambda_{1}, \lambda_{2}, \ldots, \lambda_{L}$, where $L$ is the total number of measured 3D CT data sets. For the sample described in this article, the number of CT data sets with different beam energies is $L=2$, and the number of different materials is $M=3$. Eq.(1) in [4] constitutes a linear programming problem subject to non-negativity conditions [10]. Utilizing the following material densities: $\mathrm{SrCrO}_{4}-3.9 \mathrm{~g} / \mathrm{cm}^{3}, \mathrm{TiO}_{2}-4.00 \mathrm{~g} / \mathrm{cm}^{3}$ and Polyimide $-1.43 \mathrm{~g} / \mathrm{cm}^{3}$, and assuming monochromatic beam energies at 4.5 and $6.4 \mathrm{keV}$ respectively, the coefficient matrix $A$ of eq. (1) is the following:

$$
A=\left(\begin{array}{cccc}
1 & 1 & 1 & 1 \\
0 & 1156.01 & 373.479 & 49.5686 \\
0 & 850.861 & 925.634 & 16.6585
\end{array}\right)
$$

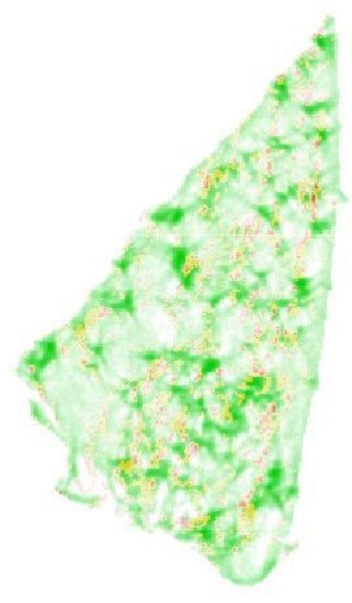

Figure 1 DCM-predicted compositional map. Strontium chromate is represented as green and titanium oxide is represented as red. The intensities represent their volume fractions respectively.

Assuming the sample contains no void, the coefficient matrix becomes

$$
A=\left(\begin{array}{ccc}
1 & 1 & 1 \\
1156.01 & 373.479 & 49.5686 \\
850.861 & 925.634 & 16.6585
\end{array}\right)
$$


The noise sensitivity of the solution is related to the condition number of the above matrix. The condition number of the matrix in Equation (2) is calculated as 8 [10]. Consequently, the maximum tolerable noise level in the input data is expected to be the order of $12 \%$. The volume fraction values are solved using the Simplex method [10] at each voxel. The solutions for all voxels form a predicted 3D compositional map of the original sample. Figure 1 shows a slice of the predicted compositional map near the middle of the sample. Quantitative comparisons of DCM predictions with image processing approaches will be the topics of future publications.

\section{Scanning electron microscopy (SEM)}

To compare the chemical composition at the surface of the sample with that computed using the data constrained model (DCM), Energy Dispersive X-ray Spectroscopy (EDX) mapping was performed on a FEI Quanta 400 field emission, environmental scanning electron microscope (ESEM) under high vacuum conditions. An electron beam energy of $15 \mathrm{kV}$ and a probe current of approximately $800 \mathrm{pA}$ were utilized, resulting in count rates of approximately $3000 \mathrm{cps}$. The electron beam was raster scanned across the surface of the sample to provide a detailed elemental map of the key constituent elements on the surface, namely $\mathrm{Ti}, \mathrm{Sr}$, and $\mathrm{Cr}$.

The sample surface imaged by the EDX process was identified in the DCM output. The DCM predicted compositional map of the surface was then projected onto an image plane parallel to the EDX sensor image plane. The EDX and DCM-projection images were then compared. Only the $\mathrm{TiO}_{2}$ and $\mathrm{SrCrO}_{4}$ compositions were used for comparison since these show well defined features in the EDX image, unlike the polymer substrate. To simulate the EDX image using DCM predicted microstructure, two alternative methods were tried: i) an averaging scheme where the image pixel intensity for each composition is proportional to the average of volume fractions within the first $C$ column voxels beneath it ( $C$ constant); ii) an attenuation scheme where a notional incident excitation beam travels down the voxel column into the material, producing a voxel dependent volume fraction response; the beam intensity is reduced as it travels through each voxel based on composition volume fractions and material properties; the voxel column ends when the beam intensity has fallen to a given fraction, nominally $1 \%$, of the incident beam, or when the beam passes completely through the sample. For the attenuation scheme, the incident beam intensity at a column voxel which is $i$ voxels away from the surface is modeled as

$$
I_{i+1}=I_{i} e^{-k \sum_{\alpha=1}^{M}\left(v_{\alpha, i} m_{\alpha} x\right) / \sum_{\alpha} m_{\alpha}} \quad ; I_{0}=1
$$

and the result $R_{\alpha}$ at each plane image pixel for each composition at the sample surface is the intensity-weighted volume fraction sum over all column voxels beneath it:

$$
R_{\alpha}=\sum_{i=0}^{C-1}\left(I_{i} \times v_{\alpha, i}\right) / \sum_{i=0}^{C-1} I_{i} .
$$

where: $I_{i}$ is the incident beam intensity at column voxel $i$ (voxel 0 is at the material surface); $m_{\alpha}$ is a density related constant for composition $\alpha$ (as a first approximation, actual composition density is used); $x$ is the (constant) thickness of each column voxel along plane normal, in units of voxels; $k$ is an empirically determined constant, units distance ${ }^{-1}$ (allows for $x$ in units of voxels and unknown $m_{\alpha}$ scaling). 
Figure 2 shows the EDX reference image and the DCM prediction derived images. There is a clear visual correlation between the DCM derived images and the EDX image. Correlation improves as voxels beneath the surface are included in the DCM derived model, but starts to degrade again as the voxel column size increases past an optimal value. In the averaging scheme, the best column height for a visual match with the EDX image was $C \approx 5$. In the attenuation scheme, the best match was found with the empirical constant $k \approx 5$. Both schemes gave similar results, with neither showing a clear advantage.

\section{Summary}

X-ray CT scans have been performed on an

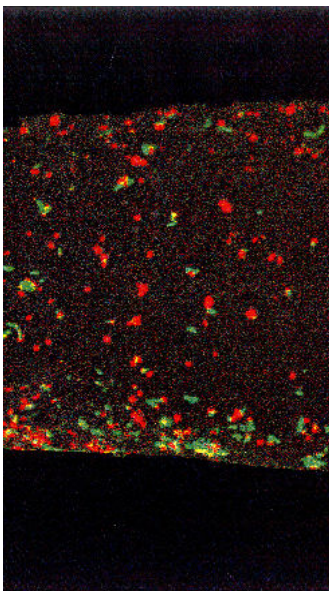

(a)

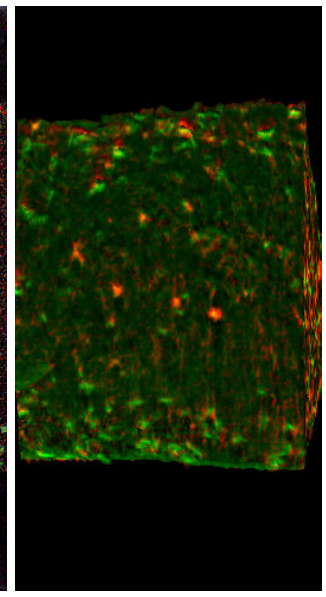

(b)

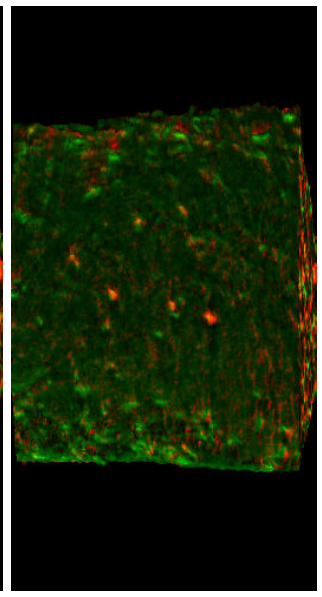

(c)

Figure 2 (a) EDX elemental map (b) DCM composition map using averaging scheme with $C=5$; (c) DCM composition map using attenuation scheme with $k=3$. $\mathrm{TiO}_{2}$ in red, $\mathrm{SrCrO}_{4}$ in green. Red and green intensities were scaled by factors 8 and 1.5 respectively to aid comparison with the EDX map. aerospace paint primer sample consisting of $\mathrm{SrCrO}_{4}$ corrosion inhibitor particles and $\mathrm{TiO}_{2}$ as $\mathrm{UV}$ resistant fillers in a polymer matrix. The DCM modeling has been used to model the microscopic compositional distributions with the X-ray CT data as model constraints. Although with a high level of experimental data errors due to X-ray equipment problems, the predicted compositional distribution on the sample surface has a promising agreement with EDX measurements. Follow-on experiments are in progress for more accurate quantitative investigations. The DCM modeling scheme is quite generic and would be used to model other systems.

\section{References}

[1] Sinko, J. (2001)Progress in Organic Coatings 42(3-4): 267-282

[2] Y. S. Yang, A. Tulloh, I. Cole, S. Furman, A. Hughes, J. Aust Ceram. Sco. 43 (2007) 159-164

[3] S. Yang, S. Furman and A. Tulloh, in Frontiers in Materials Science and Technology 2008 (Trans Tech Publications Ltd, Switzerland.UK.USA, eds. John Bell, Cheng Yan, Lin Ye and Liangchi Zhang) 267-270

[4] Y. S. Yang, T. E. Gureyev, A. Tulloh, M. B. Clennell and M. Pervukhina, Meas. Sci. Technol. 21 (2010) 047001

[5] Mayo, S.C.; Davis, T.J.; Gureyev, T.E.; Miller, P.R.; Paganin, D.; Pogany, A.; Stevenson, A.W.; Wilkins, S.W.; Opt. Express, 2003, 11, 2289-2302

[6] Mayo, S., P. Miller, D. Gao, and J. Sheffield-Parker, 2007. Journal of Microscopy-Oxford 228, 257-263

[7] Paganin, D., Mayo, S. C., Gureyev, T. E., Miller P. R.; Wilkins, S. W.; 2002, J. Microscopy, 206, $33-40$

[8] Natterer F 1989 The Mathematics of Computerized Tomography (New York: Wiley)

[9] Ibers J A and Hamilton W C 1974 eds, International Tables for X-Ray Crystallography vol 4 (Birmingham: Kynoch Press)

[10] Press W H, Teukolsky S A, Vetterling W T and Flannery B P eds, Numerical Recipes - The Art of Scientific Computing 3rd edn (Cambridge: Cambridge University Press, 2007) pp 69-73, pp $526-39$ 